\title{
Parity splitting in the alternating parity bands of some actinide nuclei.
}

\author{
R. V. Jolos ${ }^{1,2}$, and P. von Brentano ${ }^{1}$ \\ ${ }^{1}$ Institut für Kernphysik, Universität zu Köln, 50937 Köln, Germany \\ ${ }^{2}$ Bogoliubov Theoretical Laboratory, Joint Institute for Nuclear Research, 141980 Dubna, Russia
}

(May 12, 2018)

\begin{abstract}
An angular momentum dependence of the parity splitting in the alternating parity bands of nuclei with strong octupole correlations is considered basing on the model of the octupole motion in a one-dimensional potential well conserving axial symmetry. A sign reversal of the parity splitting at higher values of the angular momentum is interpreted as a result of the Coriolis coupling to $K=1$ octupole mode. New experimental data on the

spectra of the alternating parity octupole bands are used.
\end{abstract}

Typeset using REVTEX 


\section{INTRODUCTION}

The presence of strong octupole correlations in nuclei is reflected in a significant lowering of the excitation energies of the negative parity states. This phenomena was observed in different nuclei [1,2]. Among them in ${ }^{222,224,226} \mathrm{Ra}$ and ${ }^{224,226} \mathrm{Th}$ isotopes which are considered as rotating octupole-deformed systems. In these nuclei low-lying negative parity states form the alternating parity bands together with the low-lying positive-parity states connected via strong E1 transitions. It is known that such rotational bands with $I^{\pi}=3 D 0^{+}, 1^{-}, 2^{+}, \ldots$ exist in molecules. However, in nuclei in contrast to molecules these bands are disturbed: the negative-parity states are shifted up in energy with respect to the positive-parity states. Especially interesting is the angular momentum dependence of this parity splitting. At the beginning of the rotational band the positive- and negative-parity states are split in energy by several hundreds of $\mathrm{KeV}$. Even in octupole-deformed nuclei the parity splitting is 200$300 \mathrm{KeV}$. At higher values of the angular momentum the parity splitting decreases and the energies of both positive- and negative-parity states start to have approximately the rotational angular momentum dependence appropriate to a $K=0$ band. Thus, a unified alternating parity band is forming. However, not perfectly. Namely, at some value of the angular momentum the parity splitting changes sign and the negative-parity states are lowered slightly below the positive-parity states. This effect is, however, 5-10 times smaller than the positive parity splitting observed at the beginning of the rotational band. With further increase of the angular momentum the value of this negative parity splitting decreases. Thus, there are two different effects connected with parity splitting which manifest in the different ranges of the angular momentum scale. The large positive parity splitting at low angular momentum decreases with angular momentum where as the negative parity splitting which is 5-10 times smaller than the positive parity splitting appears at higher values of the angular momentum. To see both effects it is important to have the experimental data on both positive- and negative-parity rotational states up to sufficiently high values of the angular momentum $I$. Beautiful data of this kind have been obtained recently by the Liverpool group [3]. The positive parity splitting was quantitatively interpreted in the framework of the model developed by us in [4 6] for the angular momentum range available at that time. In the present paper we shall apply the model to the description of the new data of the Liverpool group. We will show also how to generalize the model to incorporate the effect of the sign reversal of the parity splitting. As was discussed in 6 the behaviour of the parity splitting from low to moderate values of $I$, where the effect is largest, can be explained in the framework of the one-dimensional model of the octupole motion conserving axial symmetry. We will show below that the smaller effect of the negative parity splitting can be explained by Coriolis coupling of the negative parity states of the $K=0$ band to the octupole modes with $K \neq 0$.

The model developed below is a phenomenological model. In principle, parity splitting can be obtained naturally in the framework of the microscopic models with parity projection. For example, see the work by the Madrid group [11], which employ selfconsistent mean field approach with parity projection. 


\section{DOUBLE MINIMUM POTENTIAL MODEL}

To explain the angular momentum dependence of the parity splitting at low $I$ the following model has been suggested in [4] []. It was assumed that at low to moderate angular momenta the parity splitting is connected with the octupole motion in a one-dimensional potential well depending on the collective variable $a_{30}$ which describes the axially symmetric octupole deformation. In nuclei with sufficiently strong octupole correlations or in octupoledeformed nuclei this potential has two minima which are symmetrically located at positive and negative values of $a_{30}$. The two minima are separated by the barrier and the transition frequency corresponds to the parity splitting in the alternating parity bands. The height of the barrier depends on the angular momentum via a rotational energy term $\frac{\hbar^{2}}{2 \Im} I(I+1)$. Due to dependence of the moment of inertia on the octupole deformation parameter $a_{30}$ [7 11] the effective barrier height between two minima increases with angular momentum $I$ proportional to $I(I+1)$ thus decreasing the probability of the barrier penetration. As a result the octupole deformation stabilizes and the parity splitting goes to zero. For physically reasonable shapes of the octupole potential it was shown that the parity splitting can be quite well described as an exponentially decreasing function of the barrier height. This result has been exploited to parameterize the angular momentum dependence of the parity splitting $\Delta E(I)$ (this quantity was introduced at first in [8]) in a simple fashion

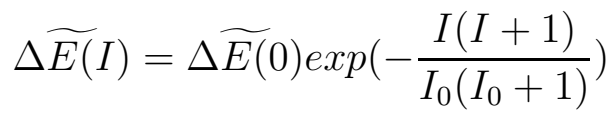

(In the previous paper [6] the notation $J_{0}$ has been used, however, instead of $I_{0}$ ) This formula has been suggested in [5]. It contains two parameters and describes a smooth decrease of the parity splitting without changing of the sign of $\widetilde{\Delta E(I)}$.

\section{CORIOLIS COUPLING TO THE INTRINSIC STATE WITH $K^{\pi}=1^{-}$}

The importance of the bands coupling effect for the description of the rotational alternating parity band was discussed in [9]. In this section we consider phenomenologically a possible effect of the Coriolis coupling of the $K^{\pi}=0^{-}$and $K^{\pi}=1^{-}$states. To do this we must have a model for the intrinsic state wave functions. A suitable formalism is the coherent state method [12,13. To use it we introduce at first creation and annihilation operators of the intrinsic phonons

$$
a_{30} \sim\left(b_{30}^{+}+b_{30}\right)
$$

The intrinsic wave functions of the $K=0$ states with even angular momentum (positive parity) and odd angular momentum (negative parity) have different parities with respect to reflection. We will take the following expressions for them

$$
\begin{aligned}
\mid \pi=(-1)^{I}, K & =0>=\frac{1}{\sqrt{2\left(\exp \left(\lambda^{2}\right)+(-1)^{I} \exp \left(-\lambda^{2}\right)\right)}} \\
& \times\left(\exp \left(\lambda b_{30}^{+}\right)+(-1)^{I} \exp \left(-\lambda b_{30}^{+}\right)\right) \mid 0>,
\end{aligned}
$$


where $b_{30} \mid 0>=0$. In (3) the parameter $\lambda$ is proportional to the octupole deformation parameter. When $\lambda$ goes to zero we get

$$
\begin{aligned}
\mid \pi=+1, K=0> & \rightarrow \quad \mid 0>, \\
\mid \pi=-1, K=0>\quad \rightarrow \quad & b_{30}^{+} \mid 0>
\end{aligned}
$$

Thus, we get the limit of the harmonic octupole vibrations. It is seen that the states (3) with even and odd $I$ have a different parity with respect to the $a_{30} \rightarrow-a_{30}$ reflection, i.e. with respect to the $b_{30}^{+} \rightarrow-b_{30}^{+}$transformation. It is easy to check and it is seen already from the limit (14) that odd angular momenta states have a larger average number of the $K=0$ octupole phonons than even angular momenta states. This circumstance is important for the consideration below. From this it follows that the Coriolis matrix elements are larger for odd angular momenta states. For very large $\lambda$ (strong octupole deformation) this difference goes to zero.

For the intrinsic states with $K=1$ we use by analogy to (3) the following expression

$$
\begin{aligned}
\mid \pi=(-1)^{I}, & K=1>=\frac{1}{\sqrt{2\left(\exp \left(\lambda^{2}\right)-(-1)^{I} \exp \left(-\lambda^{2}\right)\right)}} \\
& \times\left(\exp \left(\lambda b_{30}^{+}\right)-(-1)^{I} \exp \left(-\lambda b_{30}^{+}\right)\right) b_{31}^{+} \mid 0>,
\end{aligned}
$$

The Coriolis interaction has a standard form

$$
H_{\text {Coriolis }}=-\frac{\hbar^{2}}{2 \Im}\left(J_{+} I_{-}+J_{-} I_{+}\right),
$$

where

$$
J_{+}=\sum_{K}<K+1\left|J_{+}\right| K>b_{3 K+1}^{+} b_{3 K}
$$

It was suggested by Bohr and Mottelson [14] to use the following expression for the matrix element $\left\langle K+1\left|J_{+}\right| K\right\rangle$ in the case of the octupole excitations

$$
<K+1\left|J_{+}\right| K>=\sqrt{(3-K)(3+K+1)}
$$

We will take, however,

$$
<K+1\left|J_{+}\right| K>=\sqrt{\left(J_{0}-K\right)\left(J_{0}+K+1\right)}
$$

considering $J_{0}$ as a free parameter. However, $J_{0} \leq 3$.

Consider now a Coriolis coupling of the $K=0$ and $K=1$ states for different angular momenta. In accordance with the arguments in Section II the odd $I, K=0$ states (3) are shifted up in energy with respect to the even $I, K=0$ states by an amount of energy $\triangle \widetilde{E}(I)$ (1). We will assume that the $K=1$ phonon has an energy $\omega$. In the nuclei considered below $\omega$ is much larger than $\widetilde{\Delta E}$, however.

For every value of the angular momentum we have two states: one with $K=0$ (3) and other with $K=1$ (5). Their energies are: 0 for $K=0$ and $\omega+\Delta \widetilde{E}(I)$ for $K=1$ and even angular momenta (positive parity), and $\Delta \widetilde{E}(I)$ for $K=0$ and $\omega$ for $K=1$ and odd angular momenta 
(negative parity). Then, considering a Coriolis interaction of the $K=0$ and $K=1$ states for different $I$ we have the following energy differences between mixing states: $\omega+\Delta \widetilde{E(I)}$ if $I$ is even and $\omega-\Delta \widetilde{E(I)}$ if $I$ is odd. Thus, the energy denominators for the Coriolis mixing are somewhat smaller for odd angular momenta compared to the even angular momenta. As a result the Coriolis coupling becomes somewhat more strong for odd angular momenta states. This effect is similar to that found for odd nuclei 15, 16 where it explains a difference in the parity splitting in even $A$ and odd $A$ nuclei.

Consider now the matrix elements of the Coriolis interaction (6) between the $K=0$ (3) and $K=1$ (5) states. The square of this matrix element is proportional to an average number of the $b_{30}^{+}$phonons in the corresponding states. As it is discussed above in this section the odd angular momenta states have a larger number of the $b_{30}^{+}$phonons than even angular momenta states. Therefore the Coriolis interaction matrix elements are larger for odd angular momenta than for even angular momenta states. Together with the somewhat less important fact that the energy differences between mixing states are somewhat larger for the odd $I$ states it means that the odd $I$ states of the alternating parity band will be shifted down more than the even $I$ states. This effect can explain the observed lowering of the odd I (negative-parity) states relative to the even $I$ states, i.e. sign reversal of the parity splitting at the large values of $I$ when the Coriolis interaction becomes strong.

When the octupole deformation is stabilized $\Delta \widetilde{E(I)}$ goes to zero, the difference in the Coriolis matrix elements for odd and even $I$ states goes to zero as shown in (12) and we get an alternating parity band without staggering.

Let us introduce a quantity $\Delta E_{t h}(I)$, which describes parity splitting in the ground state alternating parity band and, in contrast to $\Delta \widetilde{E(} I)$, includes both barrier penetration and Coriolis mixing effects. Using the standard formulae for the two-level mixing we get the following expression for $\Delta E_{t h}(I)$

$$
\Delta E_{t h}(I)=\frac{\Delta \widetilde{E(}(I)-\frac{1}{\omega}\left[F_{-}^{2}(I)-F_{+}^{2}(I)\right]}{\sqrt{\frac{1}{4}\left(1+\frac{\overrightarrow{\widetilde{E(I}}}{\omega}\right)^{2}+\frac{1}{\omega^{2}} F_{+}^{2}(I)}+\sqrt{\frac{1}{4}\left(1-\frac{\Delta \widetilde{E(I)}}{\omega}\right)^{2}+\frac{1}{\omega^{2}} F_{-}^{2}(I)}}
$$

where

$$
F_{ \pm}^{2}(I)=\frac{1}{2}\left(\frac{\hbar^{2}}{\Im}\right)^{2} J_{0}\left(J_{0}+1\right) I(I+1) n_{ \pm}(I)
$$

The quantity $\Delta \widetilde{E}(I)$ introduced in the preceding section includes only an effect of the barrier penetration. Here $n_{+}(I)\left(n_{-}(I)\right)$ is an average number of the $K=0$ octupole bosons in the $I$ even (odd) states. Using the coherent states introduced above and the Hamiltonian expressed in terms of the $b_{30}^{+}, b_{30}$ boson operators describing axially symmetric octupole motion and having a potential energy term with two octupole-deformed minima we can show numerically that when octupole deformation becomes relatively stable the following approximate expressions for $n_{ \pm}(I)$ can be used

$$
n_{+}(I) \simeq n\left(1-\left[\frac{\Delta \widetilde{E(I)}}{\widetilde{E(}(0)}\right]^{b}\right)
$$




$$
n_{-}(I) \simeq\left[\frac{\Delta \widetilde{E(I)}}{\Delta \widetilde{E(0)}}\right]^{b}+n\left(1-\left[\frac{\Delta \widetilde{E(I)}}{\Delta \widetilde{E(0)}}\right]^{b}\right)
$$

with $b=0.15 \div 0.25$. The results shown below are obtained with $b=0.15$. However, when $b=0.25$ that fit is not changed essentially. In (12) $n$ is a parameter depending on the parameters of the Hamiltonian. We note that as follows from Eqs. (10 12) $\Delta E_{t h}(I) \rightarrow 0$ if $\Delta \widetilde{E(I)} \rightarrow 0$.

Now the expression (10) for the parity splitting which includes the effects of the barrier penetration and of the Coriolis mixing with the $K=1$ octupole vibrations is determined

completely. It contains the following five parameters: $\Delta \widetilde{E(0)}, I_{0}, \frac{\hbar^{2}}{\Im} \sqrt{J_{0}\left(J_{0}+1\right)}, \omega$ and $n$. In the following section we will take two of these parameters, namely, $\omega$ and $n$ to be fixed for all considered nuclei. In some nuclei the value of $\omega$ is known. $=20$

\section{DESCRIPTION OF THE EXPERIMENTAL DATA}

Since positive- and negative-parity states have different angular $=$ momenta (even and odd, correspondingly) parity splitting can be determined only by interpolation of the experimental energies of the positive-parity states to odd angular momentum and vice versa. Based only on the experimental energies we have used the following formula to determine experimental parity splitting

$$
\begin{array}{r}
\Delta E_{\text {exp }}(I)=(-1)^{I}\left(\frac{1}{2}\left[E_{\text {exp }}(I+1)-2 E_{\text {exp }}(I)+E_{\text {exp }}(I-1)\right]\right. \\
\left.-\frac{1}{8}\left[E_{\text {exp }}(I+2)-2 E_{\text {exp }}(I)+E_{\text {exp }}(I-2)\right]\right)
\end{array}
$$

if $I \geq 2$, and

$$
\begin{aligned}
\Delta E_{\text {exp }}(1)= & -\left(\frac{1}{2}\left[E_{\text {exp }}(2)-2 E_{\text {exp }}(1)\right]\right. \\
& \left.-\frac{1}{8}\left[E_{\text {exp }}(4)-2 E_{\text {exp }}(3)\right]\right)
\end{aligned}
$$

for $I=1$. We note that this formula differs slightly from the formula for $\Delta E_{\exp }(I)$ which was used in [6]. The quality of this formula can be verified by subtracting $\Delta E_{\text {exp }}(I)$ from the experimental energies $E_{\text {exp }}(I)$

$$
E_{a v}(I) \equiv E_{\exp }(I)+\frac{1}{2}(-1)^{I} \Delta E_{\exp }(I)
$$

The resulting function $E_{a v}(I)$ should be a smooth function of $I$. We have calculated $E_{a v}(I)$ for all considered nuclei and found that $E_{a v}(I)$ is really a smooth function of $I$.

We consider the following nuclei: ${ }^{220,222,224,226} \mathrm{Ra}$ and ${ }^{222,224} \mathrm{Th}$. The data are taken from [3, 18 21]. The experimental splitting $\Delta E_{\exp }(I)$ is shown in Figs. (1-6). It is compared to the theoretical splitting $\Delta E_{t h}(I)$ obtained from (10 [12). In calculating $\Delta E_{t h}(I)$ we note that the value of $\omega$ is known for ${ }^{224} \mathrm{Ra}(1053 \mathrm{KeV})$ and ${ }^{226} \mathrm{Ra}(1048 \mathrm{KeV})$. Since these two energies are quite close we have taken $\omega=1050 \mathrm{KeV}$ for all nuclei under consideration. We 
have also taken $n=3$ for all considered nuclei to reduce the number of the free parameters. This value is in a correspondence with the coherent states parameters obtained in [12]. Thus, to fit the experimental spectra we use 3 parameters for every nucleus, namely, $\Delta \widetilde{E}(0), I_{0}$ and $\frac{\hbar^{2}}{2 \Im} \sqrt{J_{0}\left(J_{0}+1\right)}$.

The results of the fit of the angular momentum dependence of the parity splitting $\Delta E_{t h}(I)$ are shown in Figs.1-6. The parameters used in the fit are presented in Table 1, where for completeness the values of the attenuation factor of the Coriolis interaction are presented. If we estimate the value of $\hbar^{2} / \Im$ fitting $E_{a v}(I)$ by the simple rotational formula $\left(E_{a v}(I+1)-\right.$ $\left.E_{a v}(I)\right)=\frac{\hbar^{2}}{\Im}(I+1)$ we get for all considered nuclei the value of $J_{0}$ not exceeding 1.1. We get the minimum value of $J_{0}=0.6$ for ${ }^{226} \mathrm{Ra}$. Thus, the values of the Coriolis strength parameter needed to fit the spectra are quite reasonable. The corresponding attenuation factor of the Coriolis interaction takes the values between 0.28 and 0.44 . We have performed also the calculations with the same attenuation factor for all considered nuclei, which we have taken to be equal to 0.33 . In this case the results for parity splitting in ${ }^{222,224} \mathrm{Ra}$ and ${ }^{224} \mathrm{Th}$ are practically coincide with those shown in Figs.2,3 and 5. The results for ${ }^{226} \mathrm{Ra}$ and ${ }^{222} \mathrm{Th}$ deviate slightly from the preceding ones. However, these deviations do not exceed $6 \mathrm{KeV}$ for ${ }^{226} \mathrm{Ra}$ and $5 \mathrm{KeV}$ for ${ }^{222} \mathrm{Th}$. The results obtained for ${ }^{220} \mathrm{Ra}$ with common attenuation factor deviate from the best fit obtained for this nucleus more than for other considered nuclei. Namely, these deviations take the values up to $20 \mathrm{KeV}$. The results obtained with common attenuation factor 0.33 for ${ }^{220,226} \mathrm{Ra}$ are presented in Figs. 1 and 4.

The value of $\Delta \widetilde{E}(0)$ characterize a stability of the octupole deformation in the ground state. This quantity is connected with the freequency of transition through the octupole barrier. The smaller the value of $\Delta \widetilde{E(0)}$, the smaller the value of the transition freequency, the larger the barrier height separating two symmetrically located octupole minima with different signs of the octupole deformation. For nuclei considered in this paper $\Delta \widetilde{E(0)}$ takes the values between $200 \mathrm{KeV}$ and $300 \mathrm{KeV}$. These values are the smallest ones among those

known for the observed alternating parity bands. In Ra isotopes the value of $\Delta \widetilde{E(0)}$ decreases initially when we go from ${ }^{226} \mathrm{Ra}$ to lighter isotopes, indicating an increase of the strength of the octupole correlations. However, it increases again in ${ }^{220} \mathrm{Ra}$. Probably, it is connected with the fact that ${ }^{220} \mathrm{Ra}$ is a transitional nucleus with $E\left(2_{1}^{+}\right)=178 \mathrm{KeV}$. In ${ }^{226} \mathrm{Ra} E\left(2_{1}^{+}\right)=68$ $\mathrm{KeV}$. The same effect is observed in Th isotopes. As is seen the largest deviations of the fit from the experimental data are obtained for ${ }^{220} \mathrm{Ra}$ and ${ }^{222} \mathrm{Th}$. These two nuclei are rather transitional ones, however. [3].

\section{CONCLUSION}

A model is developed to describe the angular momentum dependence of the parity splitting in the nuclei with strong octupole correlations. The model explains the positive parity splitting at the relatively low angular momenta and its dependence on $I$ as produced by a penetration of the barrier separating two symmetrically located octupole minima having different signs of the octupole deformation. It is demonstrated that the sign reversal of the parity splitting at higher values of $I$ can be interpreted as a result of the Coriolis mixing with $K=1$ octupole mode. However, a consideration of this effect is qualitative and a more detailed treatment requires a microscopic approach. In some nuclei parity splitting 
changes the sign in second time [17] although its absolute value decreases in average with $I$ increase. Probably, it means that the Coriolis mixing among several intrinsic states should be considered.

\section{ACKNOWLEDGMENTS}

The authors would like to express their gratitude to Dr.R.-D. Herzberg for reading the manuscript. The work was supported in part by the BMFT under contract $060 \mathrm{~K} 602 \mathrm{I}$. One of the authors (R. V. J) is grateful to the Universität zu Köln, where this work has been done, for its support. 


\section{REFERENCES}

[1] P. A. Butler and W. Nazarewicz, Rev.Mod.Phys. 68, 349 (1996).

[2] I. Ahmad and P. A. Butler, Ann.Rev.Nucl.Part.Sci. 43, 71 (1993)

[3] J. F. C. Cocks, P. A. Butler, K. J. Cann et al., Phys.Rev.Lett. 78, 2920 (1997).

[4] R. Jolos, P. von Brentano, and F. Dönau, J. Phys. G 19, L151 (1993).

[5] R. V. Jolos and P. von Brentano, Phys. Rev. C 49, R2301 (1994).

[6] R. V. Jolos and P. von Brentano, Nucl. Phys. A 587, 377 (1995).

[7] W. Nazarewicz, P. Olanders, I. Ragnarsson, J. Dudek, and G. A. Leander, Phys. Rev. Lett. 52, 1272 (1984).

[8] W. Nazarewicz and P. Olanders, Nucl. Phys. A 441, 420 (1985).

[9] W. Nazarewicz, G. A. Leander, and J. Dudek, Nucl. Phys. A 467, 437 (1987).

[10] W. Nazarewicz and S. L. Tabor, Phys. Rev. C 45, 2226 (1992).

[11] J. L. Egido and L. M. Robledo, Nucl. Phys.A 518, 475 (1990).

[12] C. E. Alonso, J. M. Arias, A. Frank, H. M. Sofia, S. M. Lenzi and A. Vitturi, in "International Conference on Perspectives for the Interacting Boson Model', Padova, Italy, 1994, eds. R. F. Casten et al.,(World Scientific, Singapore, 1994), p. 319.

[13] A.A.Raduta, Al. H. Raduta and A. Faessler, Phys. Rev. C 78, 2990 (1997).

[14] K. Neergard and P. Vogel, Nucl. Phys. A 145, 33 (1970).

[15] G. A. Leander and R. K. Sheline, Nucl.Phys. A 413, 375 (1984).

[16] D. M. Brink, B. Buck, R. Huby, M. A. Nagarajan and N. Rowley, J.Phys. G 13, 629 (1987).

[17] Ch. Briancon and I. N. Mikhailov, in "International Conference on Nuclear Structure, Reactions and Symmetries", Dubrovnik, 1986, eds. R. A. Meyer and V. Paar, (World Scientific, Singapore, 1986 ), v.1, p. 131.

[18] J. F. Smith, J. F. C. Cocks, N. Schulz et al., Phys. Rev. Lett. 75, 1050 (1995).

[19] B. Ackermann, H. Baltzer, C. Ensel et al., Nucl. Phys. A 559, 61 (1993).

[20] P. Schüler, Ch. Lauterbach, Y. K. Agarwal, J. De Boer et al., Phys. Lett. B 174, 241 (1986).

[21] B. Schwartz, M. Dahlinger, D. Habs, E. Kankeleit, D. Schwalm and R. S. Simon, MaxPlanck-Institut für Kernphysik Heidelberg, Annual Report 1987, p.72. 


\section{FIGURES}

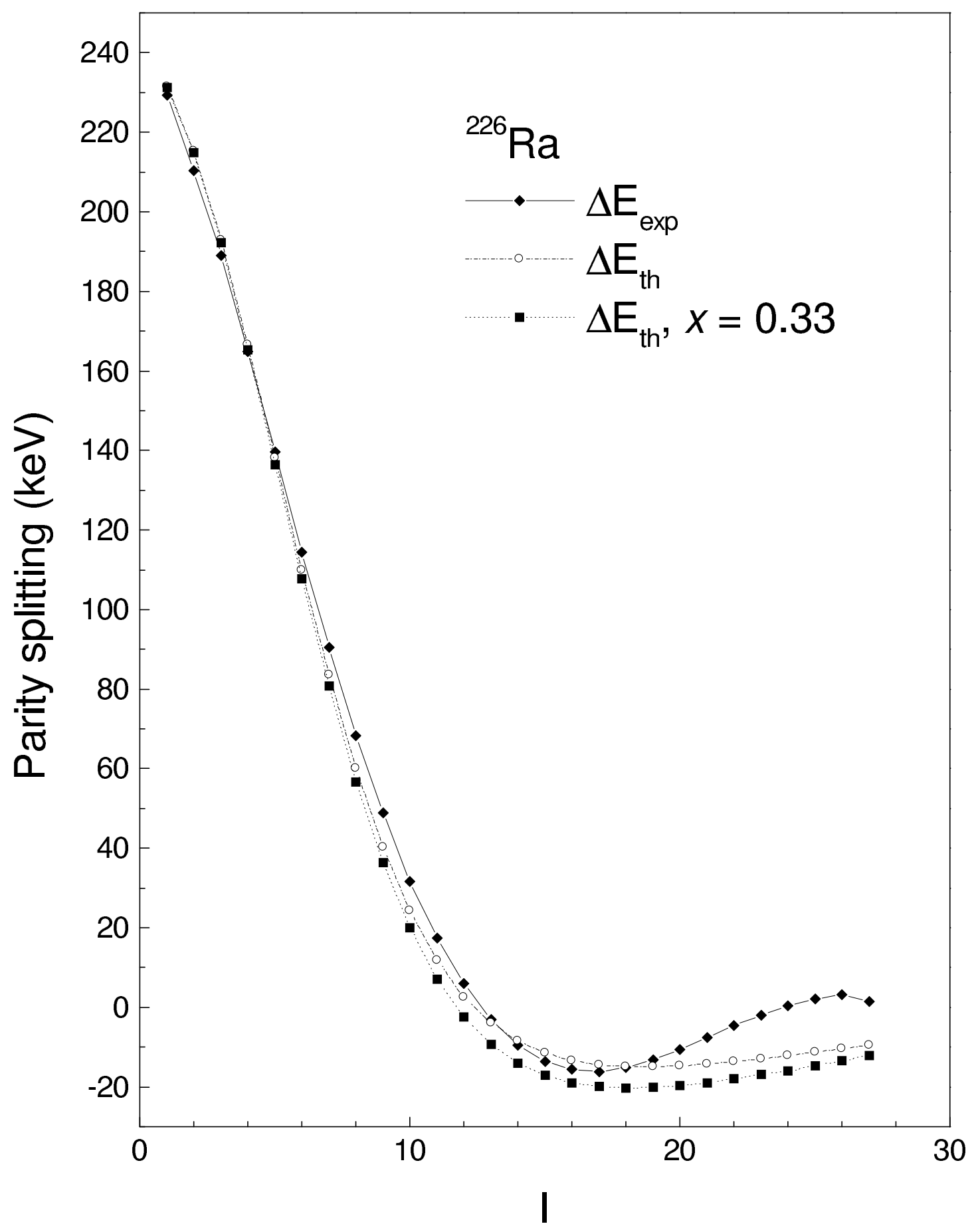

FIG. 1. Comparison of the experimental $\Delta E_{\text {exp }}(I)$ and calculated $\Delta E_{t h}(I)$ values of the parity splitting for ${ }^{226} \mathrm{Ra}$. The results of calculations obtained with the common attenuation factor for all considered nuclei $\left(\Delta E_{t h}, x=0.33\right)$ are shown in addition. The fit parameters are given in Table 1. The experimental data are taken from [3] 


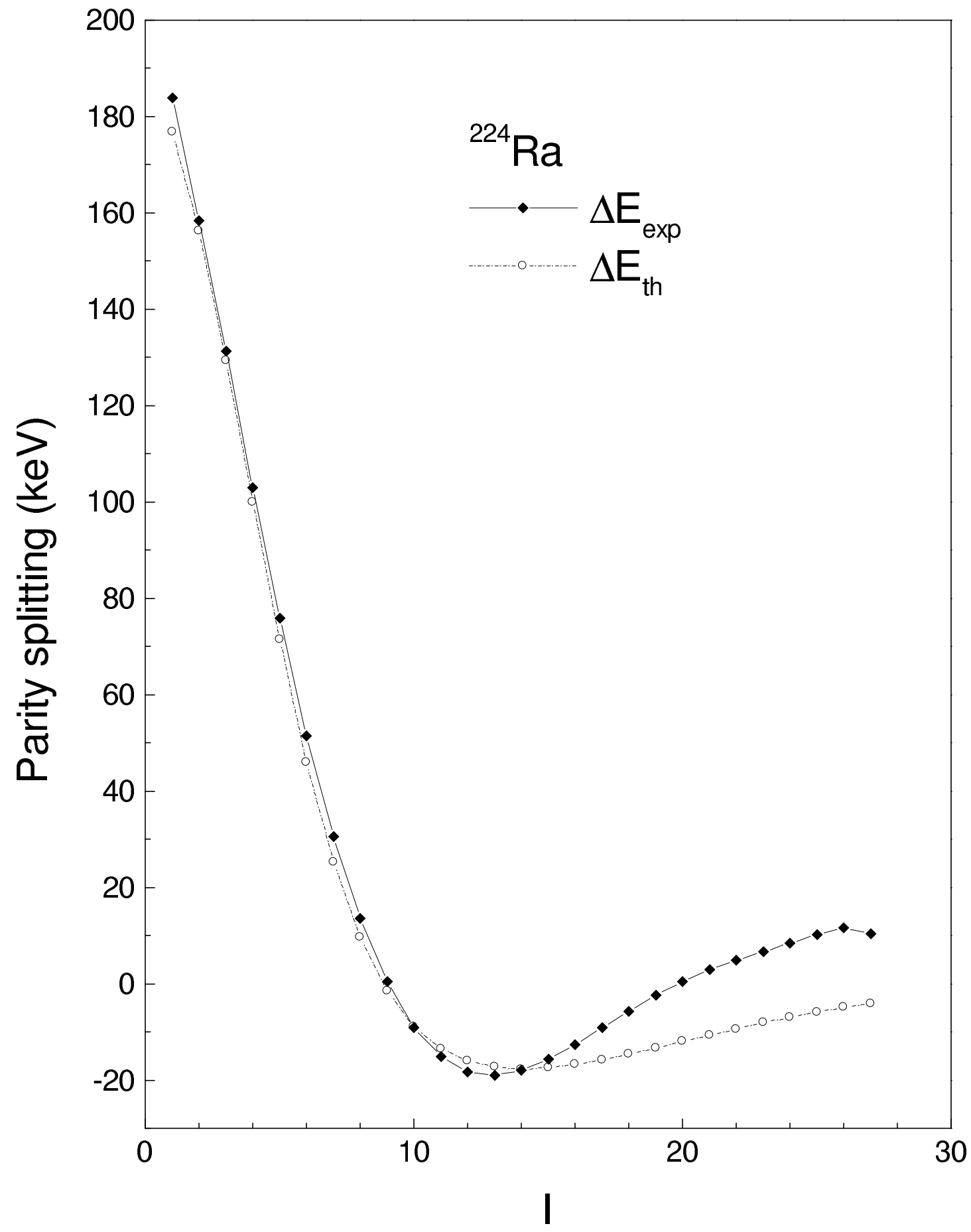

FIG. 2. Comparison of the experimental $\Delta E_{\text {exp }}(I)$ and calculated $\Delta E_{t h}(I)$ values of the parity splitting for ${ }^{224}$ Ra. The fit parameters are given in Table 1 . The experimental data are taken from $[3]$ 


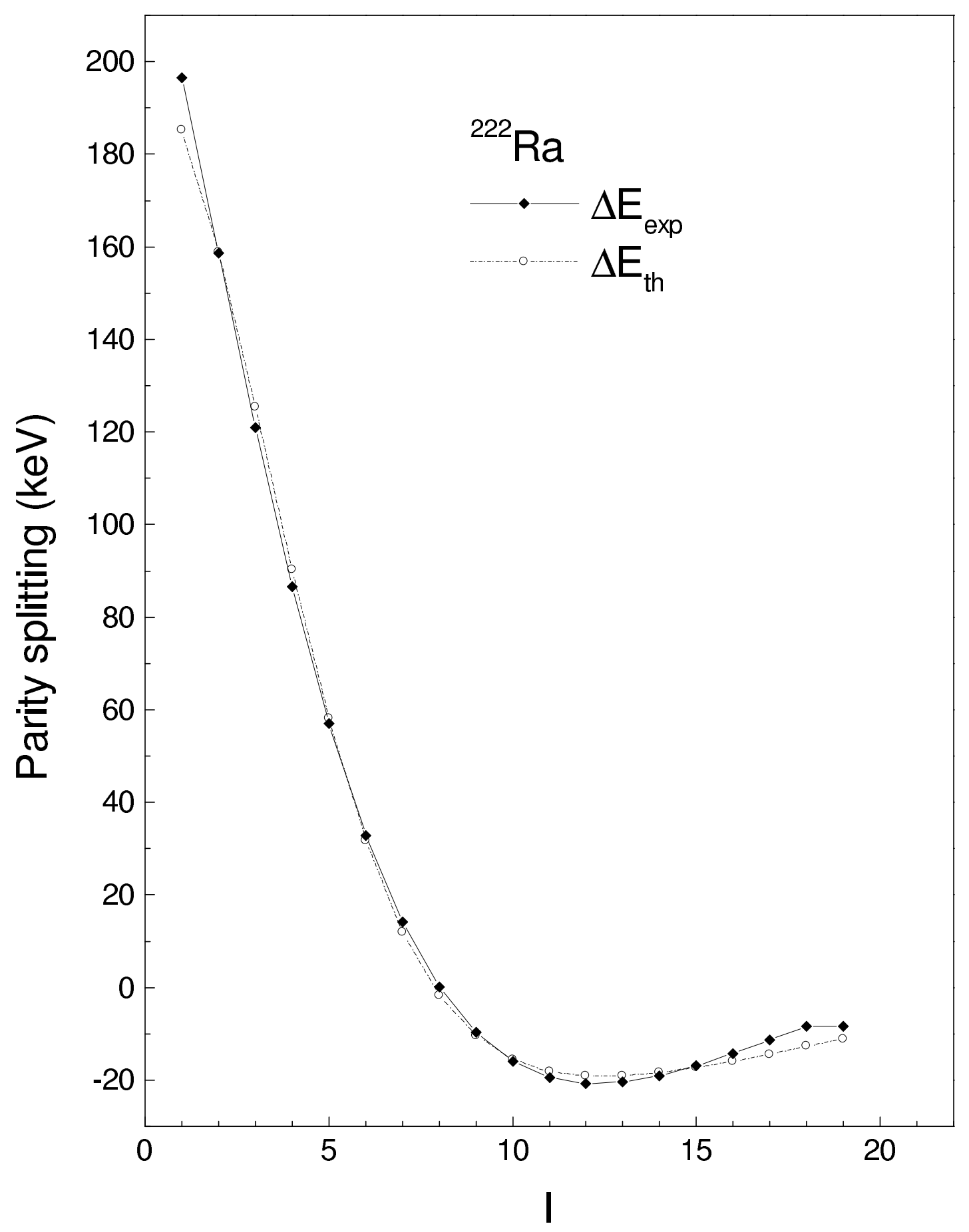

FIG. 3. The same as in Fig.2 but for ${ }^{222}$ Ra. The experimental data are taken from [3] 


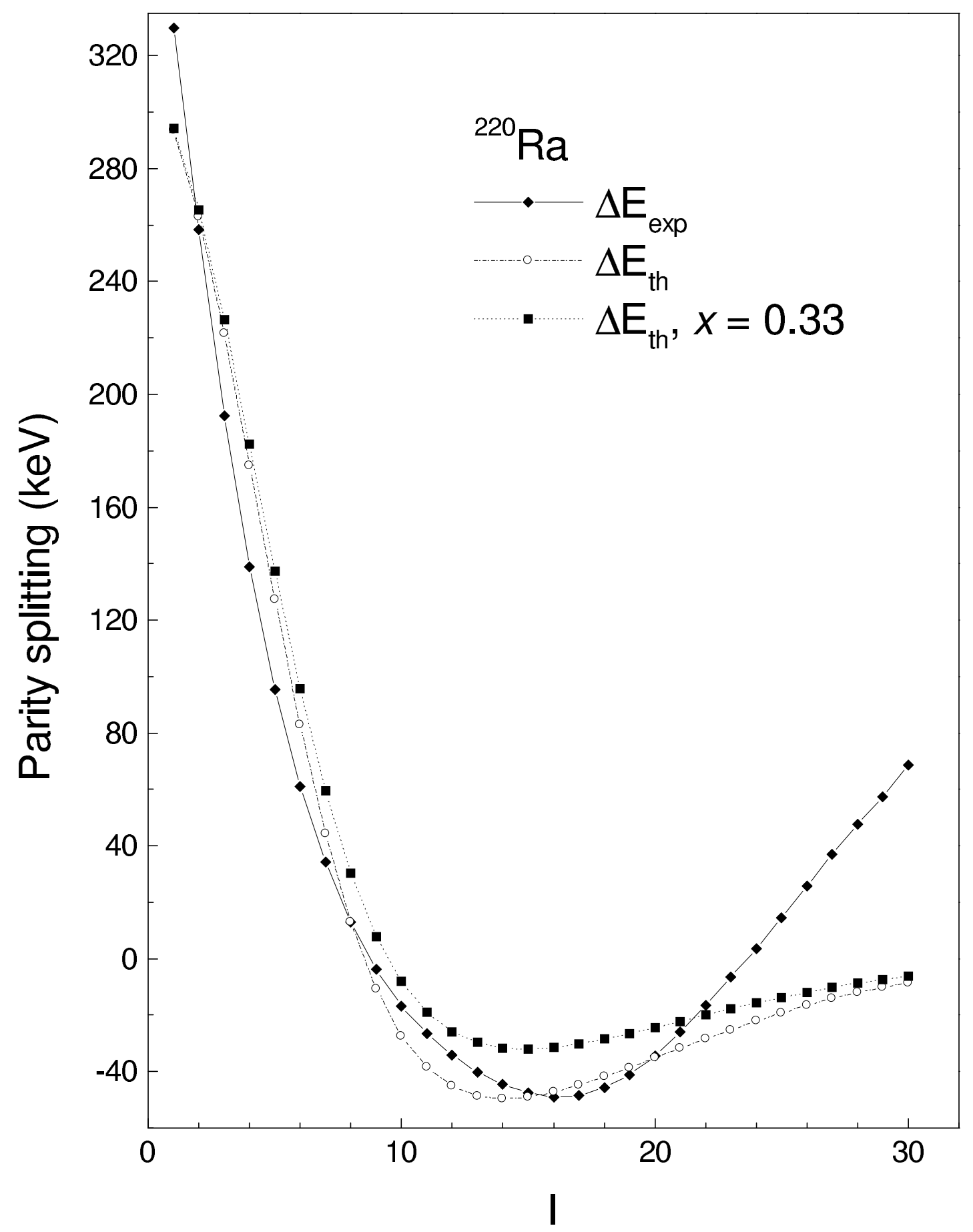

FIG. 4. The same as in Fig.1 but for ${ }^{220}$ Ra. The experimental data are taken from 18 


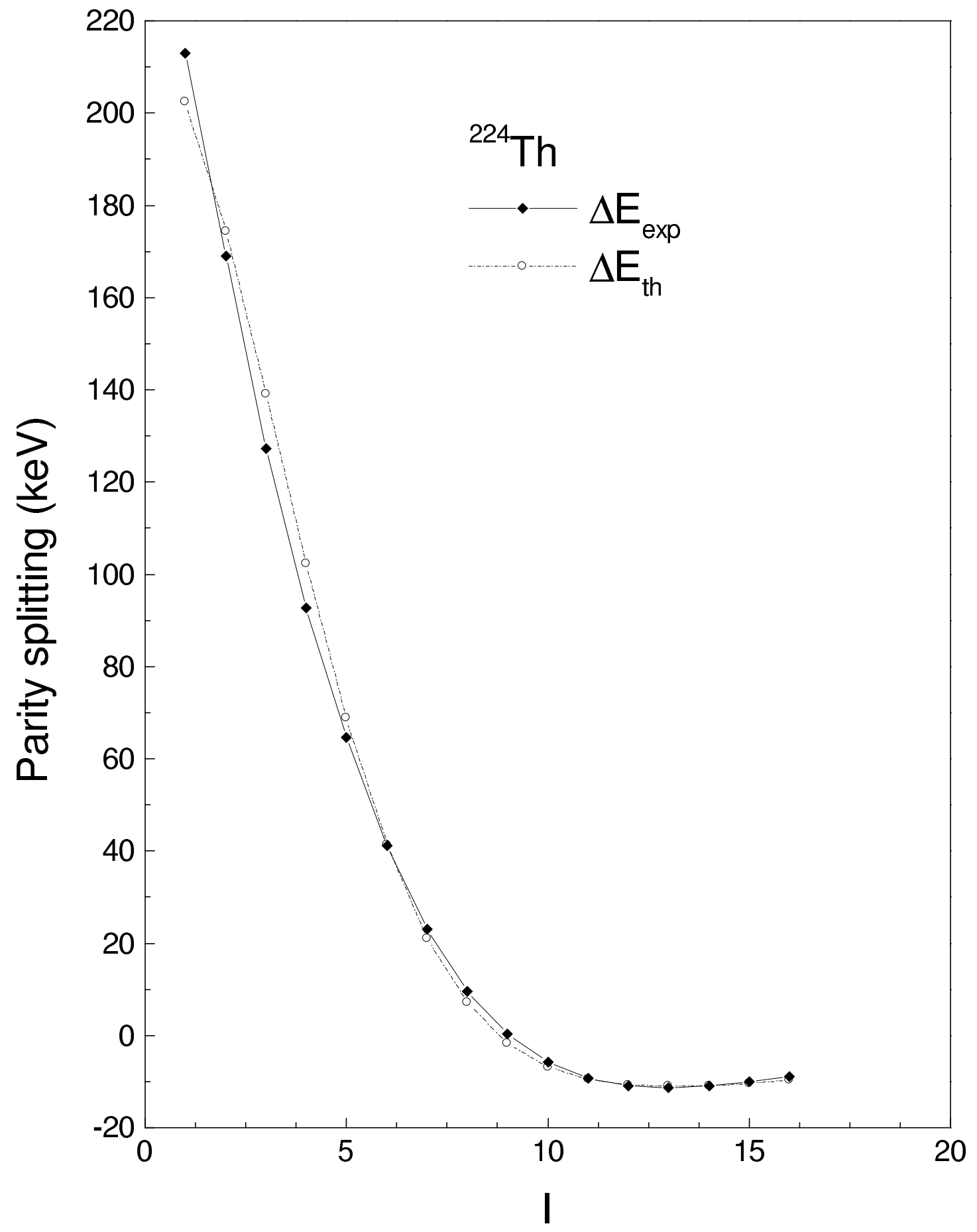

FIG. 5. The same as in Fig.2 but for ${ }^{224}$ Th. The experimental data are taken from [19,20] 


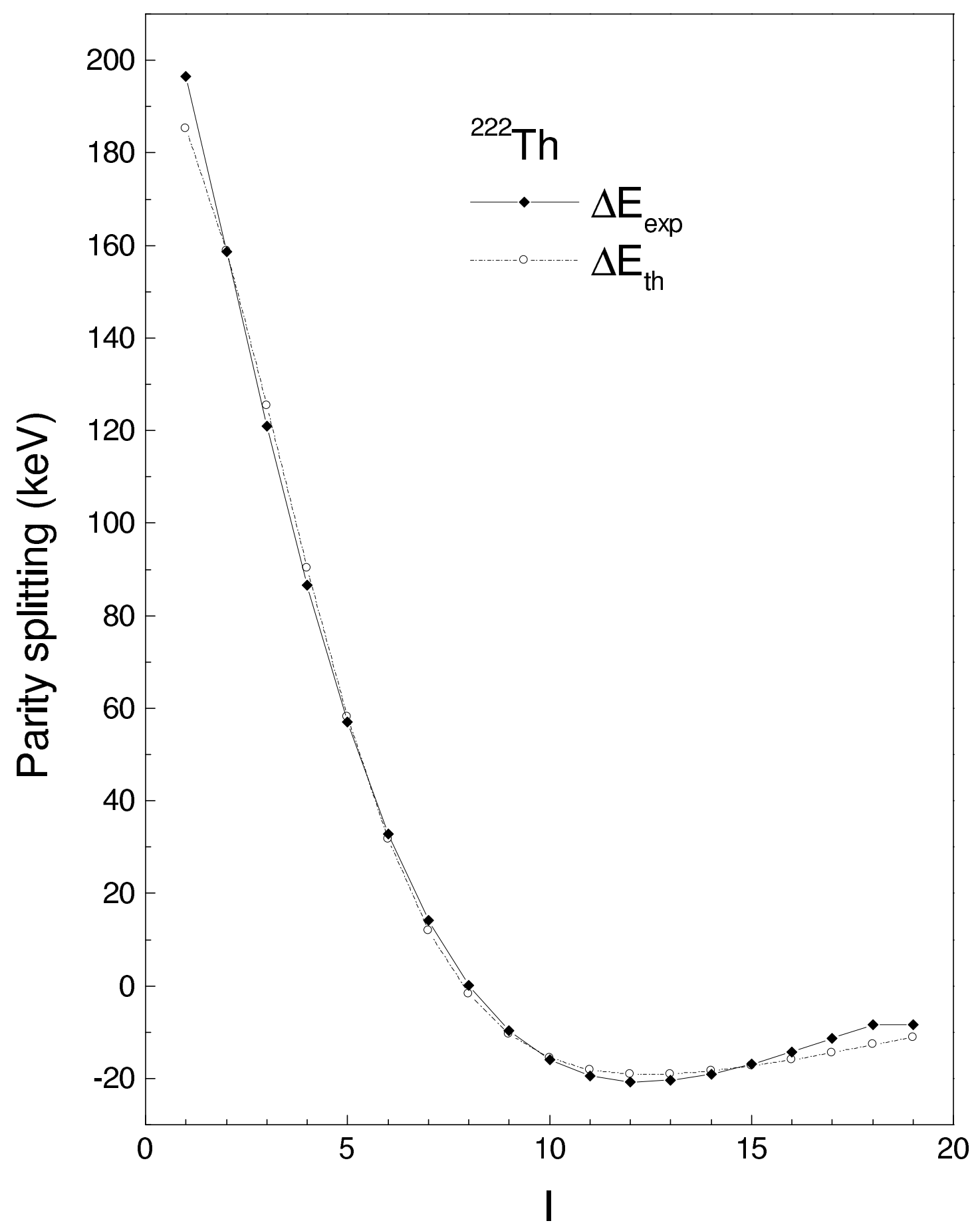

FIG. 6. The same as in Fig.2 but for ${ }^{222}$ Th. The experimental data are taken from [3,21] 


\section{TABLES}

TABLE I. Values of the parameters used for fit of $\Delta E_{\exp }(I)$ using Eqs.(10 12) and $n=3$, $\omega=1050 \mathrm{KeV}$. The values of the attenuation factor of the Coriolis interaction $x$ are shown for completeness.

\begin{tabular}{cccccc}
\hline \hline Nucleus & Ref & $\Delta \widetilde{E(0)}[\mathrm{KeV}]$ & $I_{0}$ & $\frac{\hbar^{2}}{2 \Im} \sqrt{J_{0}\left(J_{0}+1\right)}[\mathrm{KeV}]$ & $x$ \\
\hline${ }^{226} \mathrm{Ra}$ & {$[3]$} & 240 & 7.1 & 16.3 & 0.28 \\
${ }^{224} \mathrm{Ra}$ & {$[3]$} & 188 & 5.4 & 23.1 & 0.33 \\
${ }^{222} \mathrm{Ra}$ & {$[3]$} & 200 & 4.8 & 27.0 & 0.34 \\
${ }^{220} \mathrm{Ra}$ & {$[18]$} & 310 & 6.0 & 39.6 & 0.44 \\
${ }^{224} \mathrm{Th}$ & {$[19,20]$} & 218 & 4.8 & 19.8 & 0.30 \\
${ }^{222} \mathrm{Th}$ & {$[3,21]$} & 305 & 4.6 & 36.4 & 0.37 \\
\hline \hline
\end{tabular}

